\title{
XMM-Newton and Broad Iron Lines
}

\author{
A.C. Fabian ${ }^{1, \star}$ \\ Institute of Astronomy, Madingley Road, Cambridge CB3 OHA, U.K.
}

The dates of receipt and acceptance should be inserted later

Iron line emission is common in the X-ray spectra of accreting black holes. When the line emission is broad or variable then it is likely to originate from close to the black hole. X-ray irradiation of the accretion flow by the power-law X-ray continuum produces the X-ray 'reflection' spectrum which includes the iron line. The shape and variability of the iron lines and reflection can be used as a diagnostic of the radius, velocity and nature of the flow. The inner radius of the dense flow corresponds to the innermost stable circular orbit and thus can be used to determine the spin of the black hole. Studies of broad iron lines and reflection spectra offer much promise for understanding how the inner parts of accretion flows (and outflows) around black holes operate. There remains great potential for XMM-Newton to continue to make significant progress in this work. The need for high quality spectra and thus for long exposure times is paramount.

\section{Introduction}

Most of the radiation from luminous accreting black holes is released within the innermost 20 gravitational radii (i.e. $20 r_{\mathrm{g}}=20 G M / c^{2}$ ). In such an energetic environment, iron is a major source of line emission, with strong lines in the 6.4-6.9 keV band depending on ionization state. Observations of such line emission then provides us with a diagnostic of the accretion flow, conditions and the strong gravity in this regime (Fabian et al 2000; Reynolds \& Nowak 2003; Matt 2006; Miller 2007).

The rapid X-ray variability found in many Seyfert galaxies requires that the emission orginates at small radii. The high frequency break in their power spectra, for example, corresponds to orbital periods at $\sim 20 r_{\mathrm{g}}$ and variability is seen at still higher frequencies (Uttley \& McHardy 2004; Vaughan et al 2005). Key evidence that the very innermost radii are involved comes from Soltan's (1982) argument relating the energy density in radiation from active galactic nuclei (AGN) to the local mean mass density in massive black holes, which are presumed to have grown by accretion which liberated that radiation. The agreement found between these quantities requires that the radiative efficiency of accretion be 10 per cent or more (Yu \& Tremaine 2002; Marconi et al 2005). This exceeds the 6 per cent for accretion onto a non-spinning Schwarzschild black hole and inevitably implies that most massive black holes are rapidly spinning with accretion flows extending down to a just few $r_{\mathrm{g}}$. Moreover, this is where most of the radiation in such accretion flows originates.

The X-ray spectra of AGN are characterized by several components: a hard power-law which may turnover at a few hundred keV, a soft excess and a reflection component (Fig. 1). This last component is produced from sur-

\footnotetext{
^ Corresponding author: e-mail: acf@ast.cam.ac.uk
}

rounding material by irradiation by the power-law. It consists of backscattered X-rays, fluorescence and other line photons, bremsstrahlung and other continua from the irradiated surfaces. Examples of reflection spectra from photoionized slabs are shown in Fig. 2. At moderate ioniza-

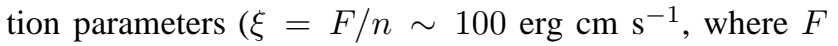
is the ionizing flux and $n$ the density of the surface) the main components of the reflection spectrum are the Compton hump peaking at $\sim 30 \mathrm{keV}$, the iron line at $6.4-6.9 \mathrm{keV}$ (depending on ionization state) and a collection of lines and reradiated continuum below $1 \mathrm{keV}$. When such a spectrum is produced from the innermost parts of an accretion disk around a spinning black hole then the outside observer sees it smeared and redshifted (Fig. 3) due to doppler and gravitational redshifts into the soft excess, the broad iron line and the Compton hump.

All three main parts of the reflection spectrum have now been seen from some AGN and Galactic Black Holes (GBH). The broad iron line and reflection hump are clearly seen in the Seyfert galaxy MCG-6-30-15 and in the GBH J1650400 (Fig. 4). More recently it has been realised that the soft excess in many AGN can be well explained by smeared reflection (Crummy et al 2005).

The extent of the blurring of the reflection spectrum is determined by the innermost radius of the disk (Fig. 4). Assuming that this is the radius of marginal stability then enables the spin parameter $a$ of the hole to be measured. Objects with a very broad iron line like MCG-6-30-15 are inferred to have high spin $a>0.95$ (Dabrowski et al 1997, Brennemann et al 2006). Some (Krolik \& Hawley 2002) have argued that magnetic fields in the disk can blur the separation between innermost edge of the disk and the inner plunge region so that the above assumption is invalid. This probably makes little difference however since the reflection comes from very dense matter. Matter in the plunge region very rapidly drops to a low density (Reynolds \& Begelman 


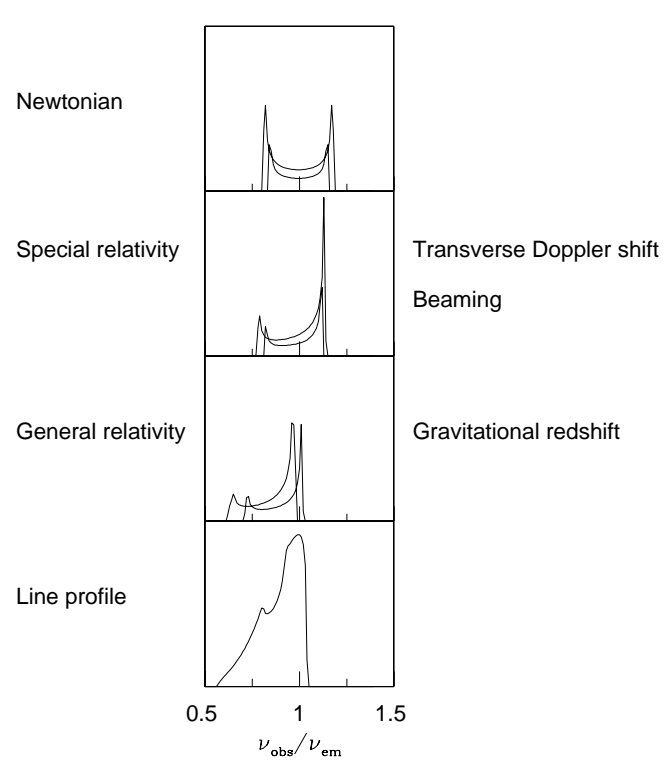

Fig. 1 The profile of an intrinsically narrow emission line is modified by the interplay of Doppler/gravitational energy shifts, relativistic beaming, and gravitational light bending occurring in the accretion disc (from Fabian et al 2000). The upper panel shows the symmetric double-peaked profile from two annuli on a non-relativistic Newtonian disc. In the second panel, the effects of transverse Doppler shifts (making the profiles redder) and of relativistic beaming (enhancing the blue peak with respect to the red) are included. In the third panel, gravitational redshift is turned on, shifting the overall profile to the red side and reducing the blue peak strength. The disc inclination fixes the maximum energy at which the line can still be seen, mainly because of the angular dependence of relativistic beaming and of gravitational light bending effects. All these effects combined give rise to a broad, skewed line profile which is shown in the last panel, after integrating over the contributions from all the different annuli on the accretion disc. Detailed computations are given by Fabian et al (1989), Laor (1991), Dovciak et al (2004) and Beckwith \& Done (2004).

1997) and only very strong fields, much larger than are inferred in disks, can stop this steep decline in density.

\section{Observations}

Broad iron lines and reflection components are seen in both AGN (e.g. Nandra et al 1997) and GBH (Miller et al 2002, 2003, 2004) with examples shown in Figs. 5, 6 and 7. They are not found in all objects or in all accretion states. There are many possible reasons for this, including overionization

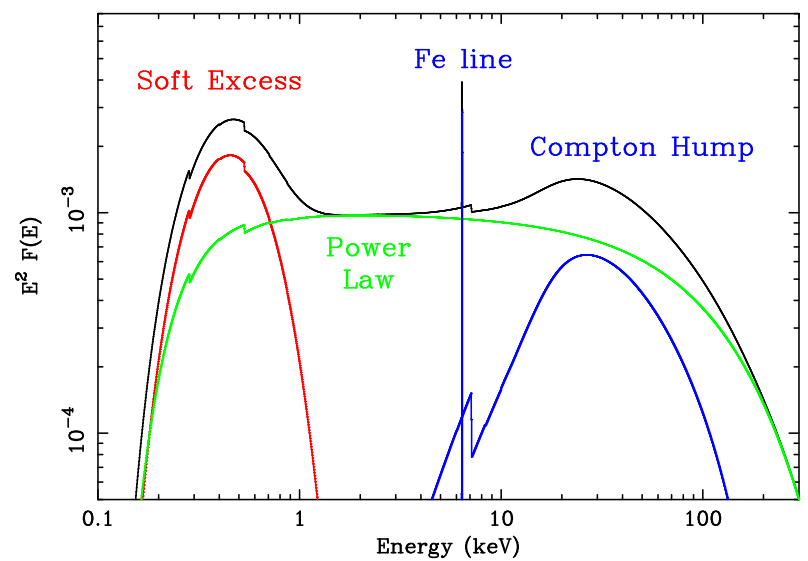

Fig. 2 The main components of the $X$-ray spectra of an unobscured accreting $\mathrm{BH}$ are shown: soft $\mathrm{X}$-ray emission from the accretion disc; power law from Comptonization of the soft X-rays in a corona above the disc; reflection continuum and narrow $\mathrm{Fe}$ line due to reflection of the hard X-ray emission from dense gas.
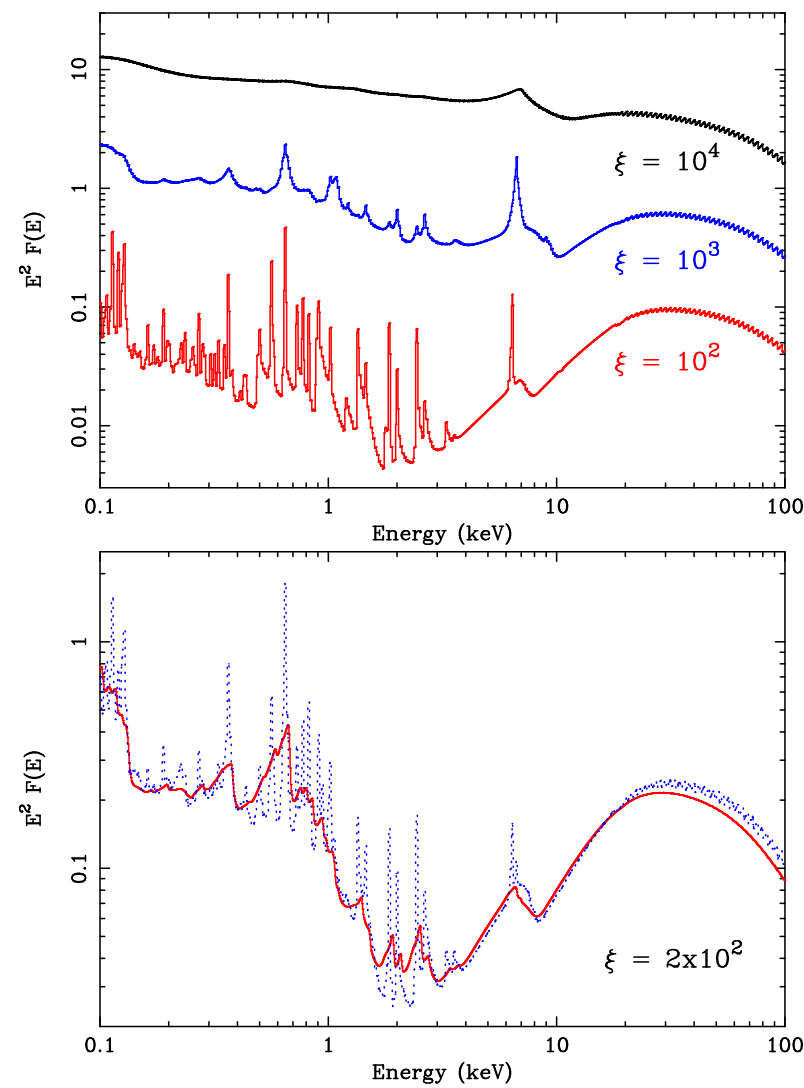

Fig. 3 Upper panel: Computed X-ray reflection spectra as a function of the ionization parameter $\xi$ ( Ross \& Fabian 2005). The illuminating continuum has a photon index of $\Gamma=2$ and the reflector is assumed to have cosmic (solar) abundances. Lower panel: Relativistic effects on the observed X-ray reflection spectrum (solid line). We assume that the intrinsic rest-frame spectrum (dotted) is emitted in an accretion disc and suffers all the relativistic effects shown in Fig. 3. 


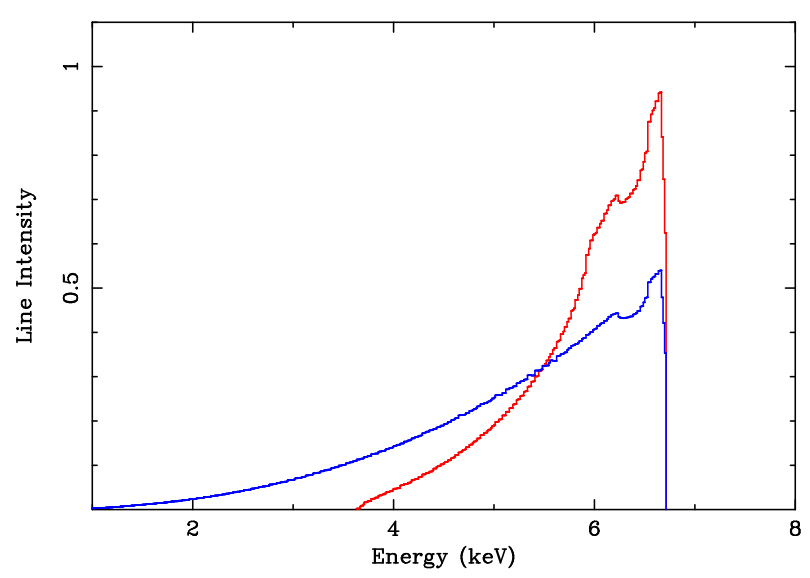

Fig. 4 The line profile dependence on the inner disc radius is shown for the two extreme cases of a Schwarzschild $\mathrm{BH}$ (red, with inner disc radius at $6 r_{g}$ ) and of a Maximal Kerr $\mathrm{BH}$ (blue, with inner disc radius at $\simeq 1.24 r_{g}$ )

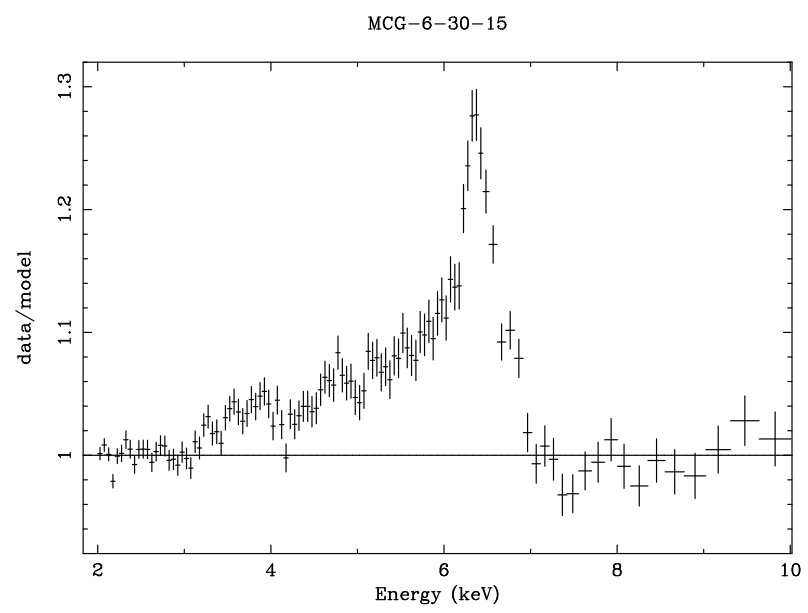

Fig. 5 The broad iron line in MCG-6-30-15 from the XMM observation in 2001 (Fabian et al. 2002a) is shown as a ratio to the continuum model.

of the surface, low iron abundance, and beaming of the primary power-law away from the disk (e.g. if the power-law originates from the mildly-relativistic base of the jet).

It should be noted that in many cases there will be a narrow iron line component due to reflection from distant matter (Fig. 8 shows an example where the narrow line dominates). Absorption due to intervening gas, warm absorbers and outflows from the AGN, as well as the interstellar medium in both our and the host galaxy must be accounted for. Moreover, if most of the emission emerges from within a few gravitational radii and the abundance is not high, then the extreme blurring can render the blurred reflection undetectable (Fabian \& Miniutti 2005).

In order to distinguish between the various spectral components, both emission and absorption, we can use higher spectral resolution, broader bandwidth and variability. An example of the use of higher spectral resolution is the work of Young et al (2005) with the Chandra high energy gratings. Observations of MCG-6-30-15 fail to show absorption

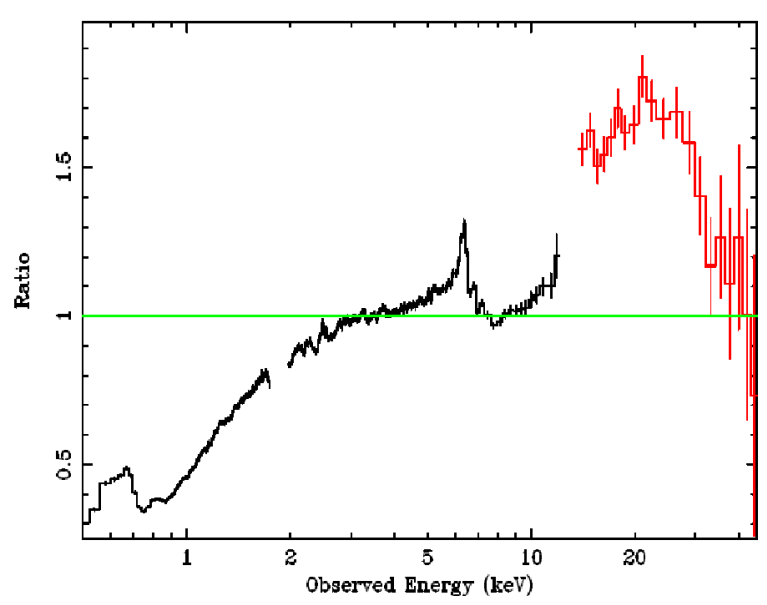

Fig. 6 Ratio of Suzaku data of MCG-6-30-15 to a powerlaw spectrum, shoing the broad iron line and the Compton hump (Miniutti et al 2007). The low energy drop is due to the warm absorber in the source.

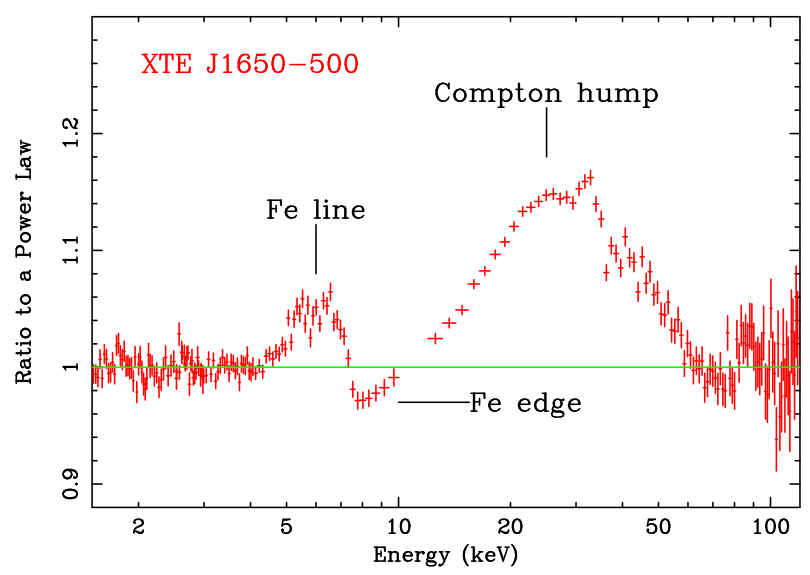

Fig. 7 Broadband BeppoSAX spectrum of XTE J1650500 (as a ratio to the continuum). The signatures of relativistically-blurred reflection are clearly seen (Miniutti et al 2004).

lines or feature associated with iron of intermediate ionization. Such gas could cause some curvature of the appearent continuum mimicking a very broad line. A broader bandwidth is very useful in determining the slope of the underlying continuum. Some work on this has been done with BeppoSAX (e.g. Guainazzi et al 1998) and Suzaku (Miniutti et al 2007; Reeves et al 2007; Markowitz et al 2007).

Broad iron lines offer the possibility for studying the effect of jet acceleration and powering on the accretion disc and flow. Some broad lines have been seen from powerful radio sources (e.g. Fig. 11; Ballantyne 2007), although much deeper exposures are required. Indeed Guainazzi et al (2006) have shown that at least 150,000 counts are needed in a typical AGN spectrum to clearly see the presence of a broad line (Fig. 10). More than 50 per cent of XMM spectra of AGN meeting that criterion do have broad lines. Nandra et al $(2006,2007)$ have shown that the incidence of broad 


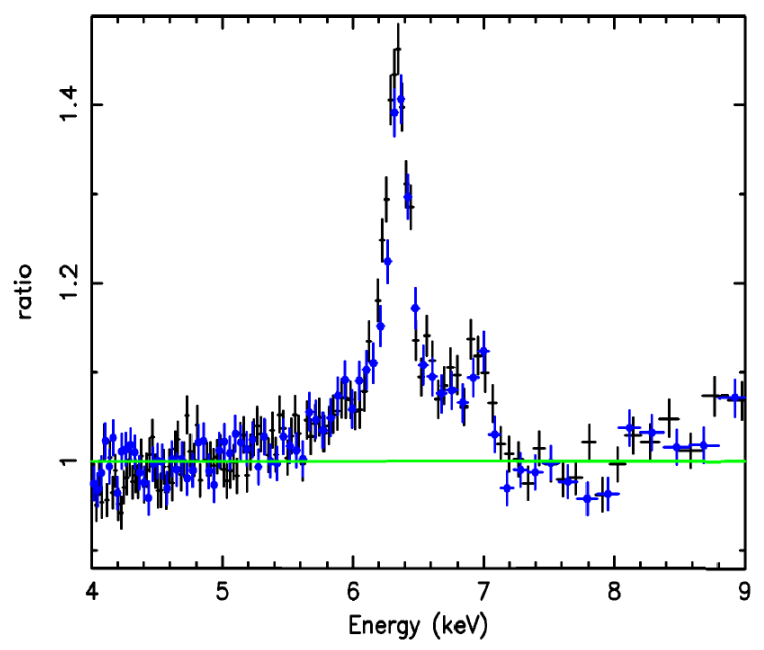

Fig. 8 Suzaku spectrum (ratio to a power-law) shoing the iron-K region. Note the intense narrow iron line at $6.4 \mathrm{keV}$, presumably from distant matter, and the broad underlying iron line from the inner disc (Reeves et al 2007).

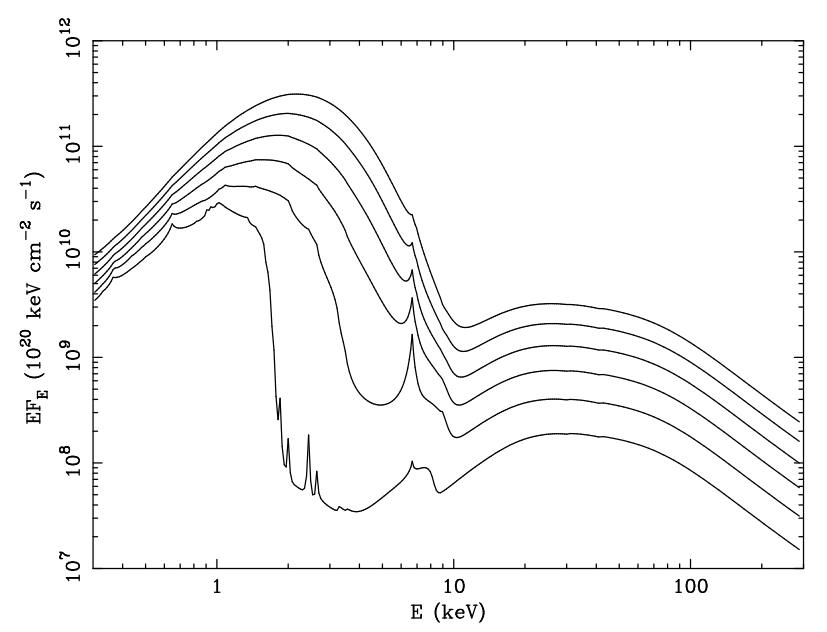

Fig.9 Irradiated disc spectra for accreting stellar mass black holes (Ross \& Fabian 2007). Thermal disc emission of increasing temperature from within the disc accounts for the large soft X-ray quasi-blackbody. The disc material is significantly hotter than for the AGN case meaning the the features undergo some Compton blurring.

iron lines in XMM AGN spectra is high, similar to that found with ASCA 10 years ago. Reports of a lack of broad iron lines in the first year or two of XMM operations (20002002) were presumably due to the relatively short exposures adopted at that time, particularly in the guaranteed observing time.

\section{Variability}

A range of variability of the iron line is seen. In some objects (e.g. NGC3516 Fig. 18, Iwasawa et al 2004; Mrk 766 Turner et al 2005) a rather narrow component is seen to

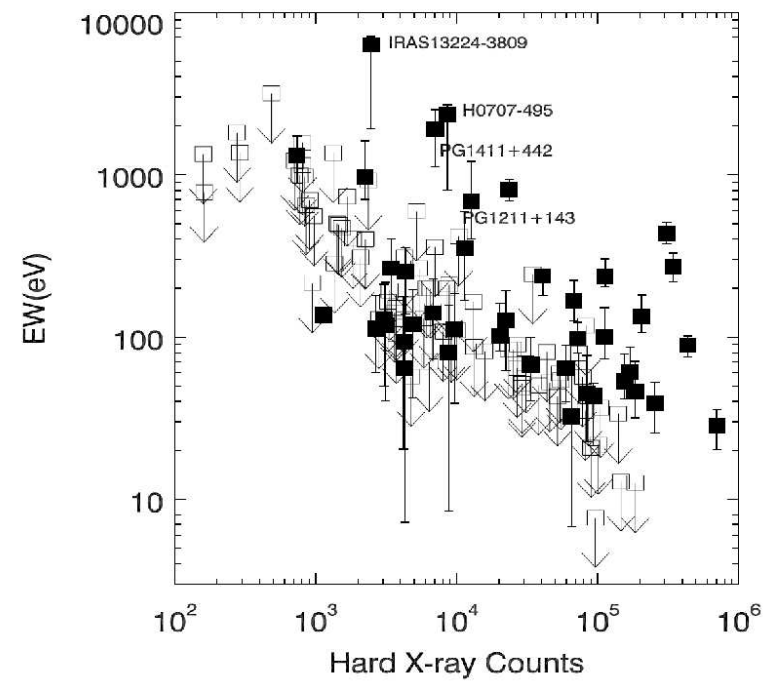

Fig. 10 Results of the analysis of XMM AGN spectra by Guainazzi et al (2006). Spectra with a broad line are shown with a filled square. More than 100,000 cts are required to clearly find a broad line in many objects.
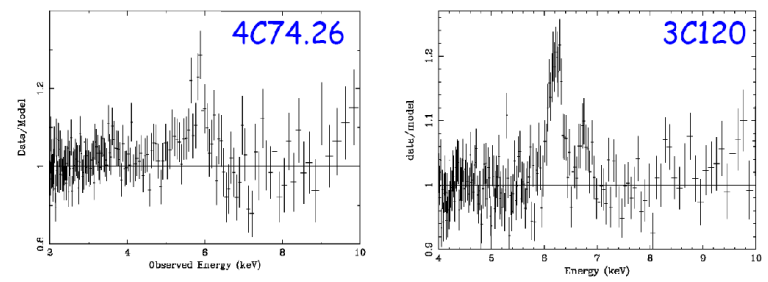

Fig. 11 XMM spectra of the iron line in the radio-loud quasar 4C 74.26 (Ballantyne et al 2006) and the broad line radio galaxy 3C 120 (Ballantyne et al 2005). See Ballantyne (2007) for a discussion.

vary in energy, possibly in a periodic manner. In others, redshifted and/or blueshifted compnents come and go (Dadina et al 2005; Turner et al 2004). In the best objects where a very broad line is seen (e.g. MCG-6-30-15 Fabian et al 2002; NGC4051 Ponti et al 2006) the reflection appears to change little despite large variations in the continuum. The spectral variability can be decomposed into a highly variable power-law and a quasi-constant reflection component. This behaviour is also borne out by a difference (high-low) spectrum which is a power-law and the spectrum of the intercept in flux-flux plots (Fig. 11).

This behaviour was initially puzzling, until the effects of gravitational light bending were included (Fabian \& Vaughan 2003; Miniutti et al 2004; Miniutti \& Fabian 2005; Suebsuwong et al 2006). Recall that the extreme blurring in these objects means that much of the reflection occurs within a few $r_{\mathrm{g}}$ of the horizon of the black hole. The enormous spacetime curvature there means that changes in the position of the primary power-law continuum have a large effect on the 


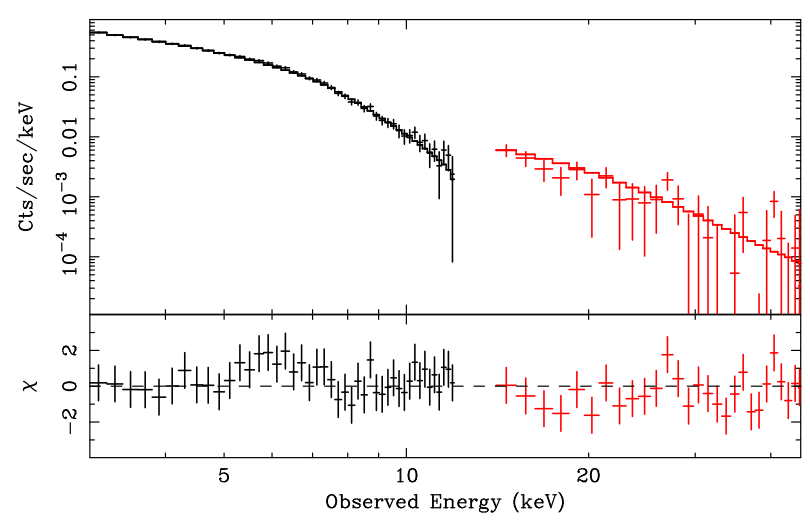

Fig. 12 Bright-faint difference spectrum for MCG-6-3015 fitted with a power-law, showing that most of the spectral variability is due to the power-law component changing in intensity (Miniutti et al 2007).

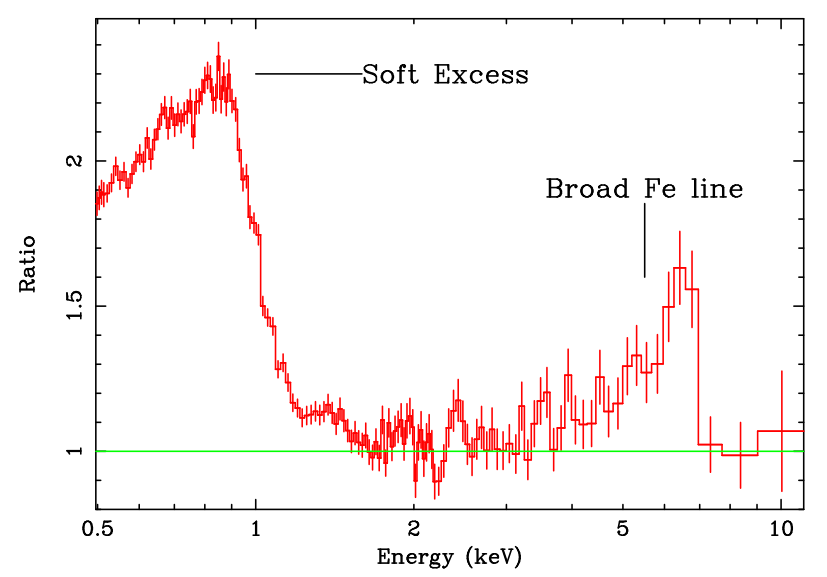

Fig. 13 Ratio of the spectrum of the NLS1 galaxy 1H0707-494 to a power-law. Spectral fits with either a very broad iron line or a partial covering with a steep edge are equally good for this object (Fabian et al 2004; Boller et al 2002).

flux seen by an outside observer (Fig. 13; Martocchia \& Matt 1996). What this means is that an intrinsically constant continuum source can appear to vary by large amounts just by changing position in this region of extreme gravity. The reflection component, however, will appear to be relatively constant in flux in this region. Consequently, the observed behaviour of these objects may just be a consequence of strong gravity. Rapid motion of the power-law source can, by special-relativistic beaming, also introduce anisotropy in the irradiation (Reynolds \& Fabian 1997; Beloborodov 1999) which contribute to the effects seen.

Some of the Narrow-Line Seyfert 1 galaxies such as 1H0707 (Fig. 14), IRAS13224 and 1H0439 appear to share this behaviour (Fabian et al 2002, 2004). Some of these objects can show sharp drops around $7 \mathrm{keV}$ that may be interpreted as due to absorption from something only partially covering the source. (If the covering was total then no strong soft emission would be seen, contrary to observation.) However the source is rapidly variable while being partially cov-

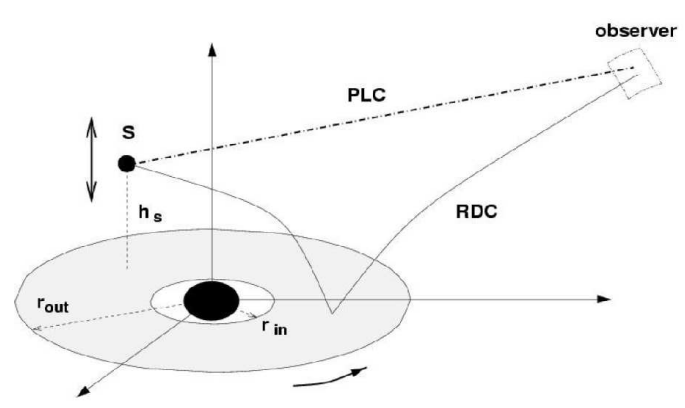

Fig. 14 Schematic of the light paths from source $S$ at height $h$ close to the black hole. The direct power-law component (PLC) and the reflection-dominated component (RDC) are indicated.

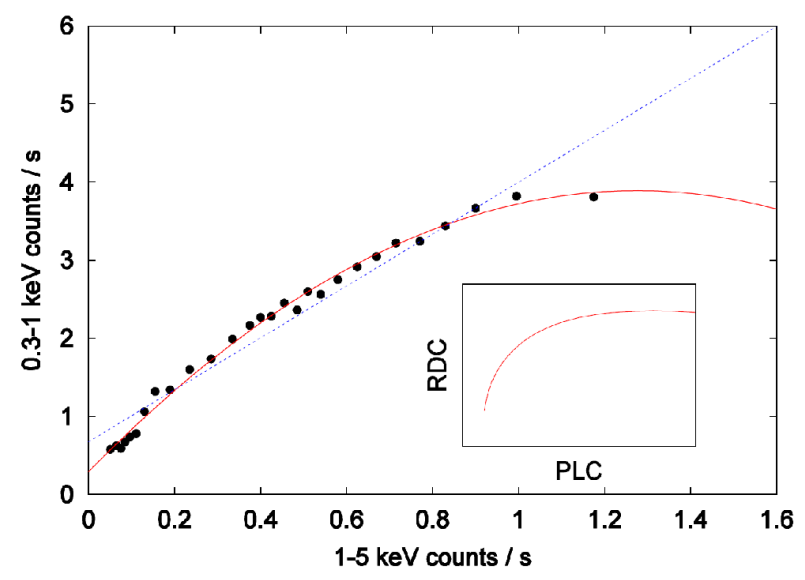

Fig. 15 Flux-flux plot for $1 \mathrm{H} 0707$. The $1-5 \mathrm{keV}$ flux on the $\mathrm{x}$-axis is dominated by the PLC, whereas the $0.3-1 \mathrm{keV}$ flux on the y-axis is dominated by reflection (the RDC). The inset shows the behaviour expected from the light bending model.

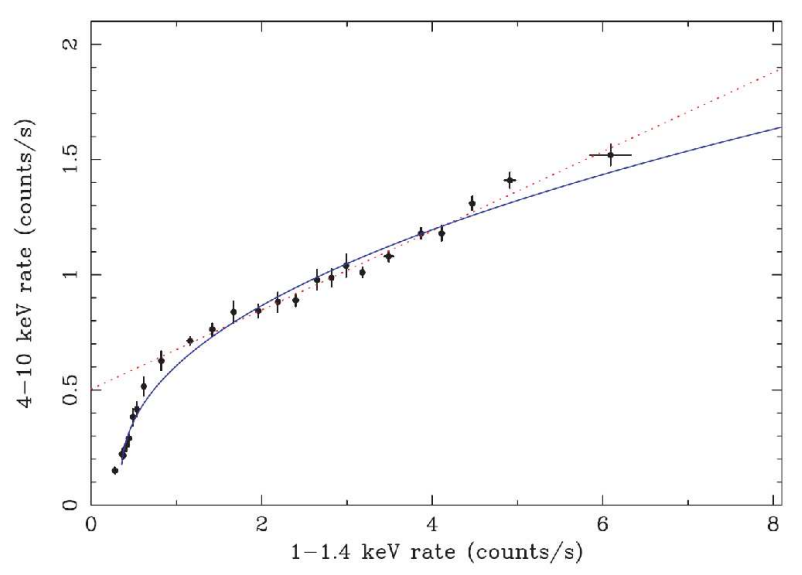

Fig. 16 Flux-flux plot for NGC 4051 (Ponti et al 2006), analagous to Fig. 15. 


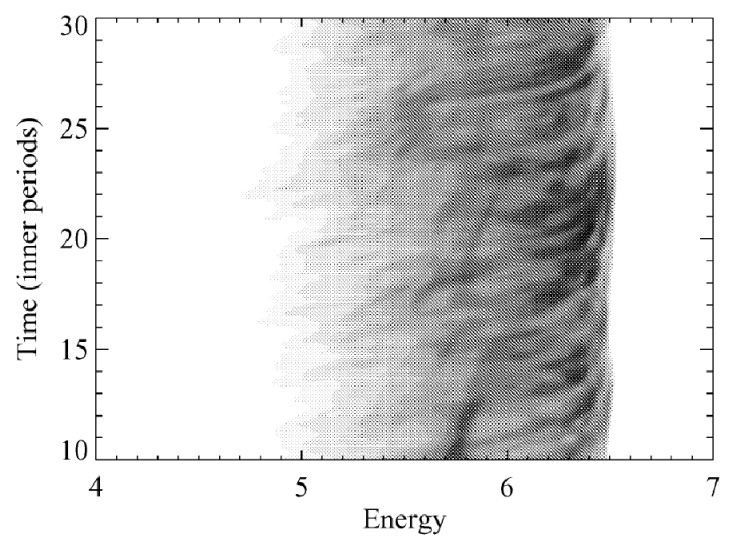

Fig. 17 Line variability expected from a turbulent accretion disc (Armitage \& Reynolds 2003).

ered which places what may be impossible constraints on what is doing the covering. Also the GBH XTEJ1650-500 behaved in a manner similar to that expected from the light bending model (Rossi et al 2005).

The important point is that the X-ray spectra of many AGN have a hard, blurred, reflection component which varies little in intensity and is in shape consistent with reflection from the innermost part of an accretion disc around a rapidly spinning black hole.

\section{Discussion}

Clear examples of relativistically-broadened iron lines are seen in some AGN and GBH in some states. Such objects must have dense inner accretion disks in order that the gas is not overionized. Detection of a line is helped greatly if the iron abundance is super-Solar and if there is little extra absorption due to very strong warm absorbers or winds. Where broad lines are seen and can be modelled satisfactorally then the spin of the central black hole can be reliably determined.

The study of absorption and emission variability of iron$\mathrm{K}$ lines is in its infancy, with some interesting and tantalising results produced so far. The inner regions of accretion flows are bound to be structured and so give rise to variations (e.g. Armitage \& Reynolds 2003). Some may be due to motion or transience in the corona or primary power-law source while others may reflect structure, e.g. spiral waves, on the disk itself, intercepting primary radiation from much smaller radii.

What XMM-Newton, and Suzaku, have established is the reality and relative ubiquity of broad iron lines and other blurred reflection features. We have a tool with which to study the inner workings of the ultimate powerhouse in the Universe, the accreting black hole. To examine variability on the light crossing time of the innermost orbits of the black holes in the bright $\mathrm{AGN}$ is beyond XMM, since squaremetre-class collecting area is required, but there are enough

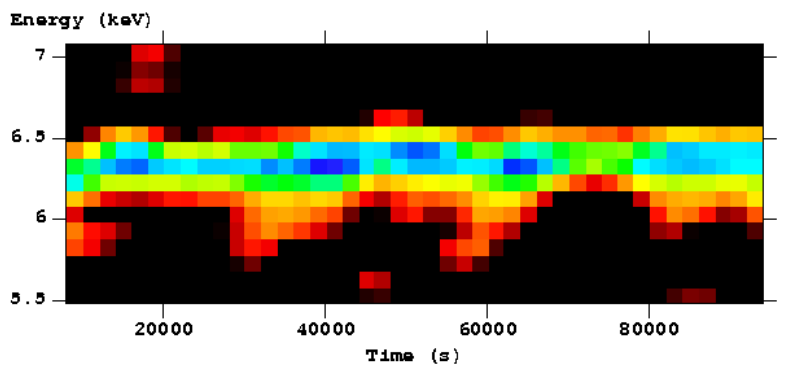

Fig. 18 Iron line variability, suggestive of periodic behaviour, see in an XMM observation of NGC 3516 (Iwasawa et al 2004).

hints from the existing patchy observations for a) the problem to be tackled in a statistical way, b) more black hole spins to be well established, c) the inner regions of jetted sources to be explored, and d) the position and/or motion of the power-law source to be tracked. We need to map how the energy is extracted from the accretion flow and deposited into the power-law generating corona, presumably by magnetic fields. This is not a minor process but accounts for at least 12 per cent of the accretion power in high Eddington ratio sources and 50 per cent or so in the low Eddington ones $\left(L_{\mathrm{Bol}} / L_{\mathrm{Edd}}<0.1\right.$; Vasudevan \& Fabian 2007).

This requires us to make much deeper exposures on many more suitable AGN, to push them over the $150,000 \mathrm{ct}$ boundary, an approach being pursued by Matteo Guainazzi and colleagues. This does of course mean long exposures on the next fainter level of sources. I favour very long exposures on the brightest sources. Ms exposures on MCG-6-30-15, for instance, or of the brightest NLS1s such as $1 \mathrm{H} 0707$ or IRAS 13224. We are only just beginning to explore these objects and parameter space is ripe for serendipitous discovery as much as nailing down the parameters we expect. As a community, we owe it to the legacy of XMM-Newton to propose, accept and follow through such work which will be invaluable for understanding the most powerful quasisteady sources in the Universe. It cannot be done any other way!

Acknowledgements. I am grateful to many colleagues for collaboration and discussion on broad iron lines, including Giovanni Miniutti, Jon Miller, Chris Reynolds, Andy Young, Randy Ross, Luigi Gallo, Josefin Larsson, Gabrielle Ponti and others. This is a heavily modified version of my contribution for the XMM meeting on Broad Iron Lines 2006.

\section{References}

Armitage P.J., Reynolds C.S., 2003, MNRAS, 341, 1041

Ballantyne D.R., 2007, astro-ph/0707.3142

Ballantyne D.R., Fabian A.C., 2005, ApJ, 622, L97

Ballantyne D.R., Fabian A.C., Iwasawa K., 2004, MNRAS, 354, 839

Beckwith K., Done C., 2004, MNRAS, 352, 353

Beloborodov A.M., 1999, ApJ, 510, L123

Boller Th. et al, 2002, MNRAS, 329, L1 
Brenneman L., Reynolds C.S., 2006, ApJ, 652, 1028

Crummy J., Fabian A.C., Gallo L., Ross R.R., 2006, MNRAS, 365, 1067

Dabrowski Y., Fabian A.C., Iwasawa K., Lasenby A.N., Rynolds C.S., 1997, MNRAS, 288, L11

Dadina M., Cappi M., Malaguti G., Ponti G., De Rosa, A., 2005, A\&A, 442, 461

Dovčiak M., Karas V., Yaqoob T., 2004, ApJSS, 153, 205

Fabian A.C., Rees M.J., Stella L., White N.E., 1989, MNRAS, 238,729

Fabian A.C., Iwasawa K., Reynolds C.S., Young A.J., 2000, PASP, 112,1145

Fabian A.C., et al. 2002a, MNRAS, 335, L1

Fabian A.C., Ballantyne D.R., Merloni A., Vaughan S., Iwasawa K., Boller Th., 2002b, MNRAS, 331, L35

Fabian A.C., Vaughan S., 2003, MNRAS, 340, L28

Fabian A.C., Miniutti G., Gallo L., Boller Th., Tanaka Y., Vaughan S., Ross R.R., 2004, MNRAS, 353, 1071

Fabian A.C., Miniutti G., Iwasawa K., Ross R.R., 2005, MNRAS, 361,795

Fabian A.C., Miniutti G., 2005, astro-ph/0507409

Guainazzi M., et al 1999, A\&A, 341, L27

Guainazzi M., et al 2006, AN, 327, 1028

Iwasawa K., Miniutti G., Fabian A.C., 2004, MNRAS, 355, 1073

Krolik J.H., Hawley J.F., 2002, ApJ, 573, 754

Laor A., 1991, ApJ, 376, 90

Marconi A., et al 2005, MNRAS, 351, 169

Markowitz A., et al 2007, preprint

Martocchia A., Matt G., Karas V., 2002, A\&A, 383, L23

Matt G., 2006, AN, 327, 949

Miller, J.M., et al. 2002a, ApJ, 570, L69

Miller, J.M., et al. 2002b, ApJ, 577, L15

Miller, J.M., et al. 2002c, ApJ, 578, 348

Miller, J.M., et al. 2003, MNRAS, 338, 7

Miller, J.M., et al. 2004a, ApJ, 601, 450

Miller, J.M., et al. 2004b, ApJ, 606, L131

Miller J.M., Fabian A.C., Nowak M.A., Lewin W.H.G., Proc. of the 10th Marcel Grossman Meeting, 20-26 July 2003, Rio de Janeiro, Brazil, astro-ph/0402101

Miller, J.M., 2007, ARAA, 45, astro-ph/07050540

Miniutti G., Fabian A.C., Goyder R., Lasenby A.N., 2003, MNRAS, 344, L22

Miniutti G., Fabian A.C., 2004, MNRAS, 349, 1435

Miniutti G., Fabian A.C., Miller J.M., 2004, MNRAS, 351, 466

Miniutti G., Fabian A.C., 2005, submitted to MNRAS

Miniutti G. et al 2007, PASJ, 59, S315

Nandra K., George I.M., Mushotzky R.F., Turner T.J., Yaqoob T., 1997a, ApJ, 476, 70

Nandra K., et al 2006, AN 327, 1039

Nandra K., et al 2007, MNRAS in press, astro-ph/0708.1305

Ponti G. et al, 2006, MNRAS, 282, L53

Reeves J. et al 2007, PASJ. 59, S301

Reynolds C.S., Begelman M.C., 1997, ApJ, 488, 109

Reynolds C.S., Fabian, A.C., 1997, MNRAS, 290, L1

Reynolds C.S., Nowak M.A., 2003, PhR, 377, 389

Ross R.R., Fabian A.C., 2005, MNRAS, 358, 211

Ross R.R., Fabian A.C., 2007, MNRAS, in press, astroph.0709.0270

Rossi S., Homan J., Miller J.M., Belloni T., 2005, MNRAS, 360, 763

Soltan A., 1982, MNRAS, 200, 115

Suebsuwong, T., Matzac J., Jourdain E., Marcowith A., 2006, A\&A, 453, 773

Turner T.J., Kraemer S.B., Reeves J.N., 2004, ApJ, 603, 62
Turner, T.J., Miller L.J., George I.M., Reeves, J.N., 2006, A\&A, 445, 59

Uttley P., McHardy I.M., 2004, Progr. Th. Phys. S155, 170

Vasudevan R., Fabian A.C., 2007, MNRAS submitted, astro$\mathrm{ph} / 0708.4308$

Vaughan S., Fabian A.C., 2004, MNRAS, 348, 1415

Vaughan S., Iwasawa K., Fabian A.C., Hayashida K. 2004, MNRAS, 356, 524

Young A.J., Lee J.C., Fabian A.C., Reynolds C.S., Gibson R.R., Canizares C.R., 2005, ApJ, 631, 733

Yu Q., Tremaine S., 2002, MNRAS, 335, 965 